\title{
The Earth's core parameters as seen by the VLBI
}

\author{
S. B. Lambert and V. Dehant
}

Royal Observatory of Belgium, 1180 Brussels, Belgium

e-mail: [s.lambert; . dehant]@oma.be

Received 2 March 2007 / Accepted 4 April 2007

\section{ABSTRACT}

\begin{abstract}
Context. From the VLBI nutation series, one can derive information on the fluid core, such as the resonant frequency associated with the retrograde free core nutation (RFCN). This frequency has already been determined, along with other parameters, and then later confirmed with a longer data set.

Aims. This paper looks at the sensitivity of the RFCN frequency with respect to three factors not considered before: the state-ofthe-art VLBI analysis strategy, possible change of the RFCN period with time, and the effects of the atmosphere contaminating the determination.

Methods. Using several geodetic VLBI nutation data sets, we estimate amplitudes of various forced nutations, from which we deduce the resonant RFCN frequency consisting in the real frequency and a damping factor. The time stability is checked by using a sliding window. The atmospheric contribution is assessed using the 6-h NCEP/NCAR Reanalysis data.

Results. We show that the resonant period is stable within half a day from one VLBI data set to another. The atmospheric angular momentum contribution is found to have no noticeable effect. However, we point out that, although small, inconsistencies between VLBI data sets do show up and could have an impact, if not corrected, in future determination of geophysical parameters, if one wants to reach a higher accuracy than at present.
\end{abstract}

Key words. reference systems - Earth

\section{Introduction}

The computation of nutation angles derived from very long baseline interferometry (VLBI) measurements against the non rigid Earth nutation model of Mathews et al. (2002) (referred to as MHB in the following) shows differences of about 100 microarc seconds ( $\mu$ as) in rms. The accuracy in the frequency domain varies from $100 \mu$ as for long-period nutations down to $10 \mu$ as for shorter ones. These residuals contain unmodeled or mismodeled terms, as well as effects due to the observational and analysis strategies (e.g., Dehant et al. 2003). Exploration of these residuals is at least expected to give information on the accuracy of any determination of the Earth's interior parameters.

The retrograde free core nutation (RFCN) is a free rotational mode of the Earth, associated with the ellipsoidal liquid core rotating inside the visco-elastic mantle. The signature of this free mode on the Earth's figure axis, observed from a space-fixed reference frame, is a retrograde motion (opposite the Earth's rotation) that reaches an amplitude of about $200 \mu$ as, variable in time, and with a variable phase (see for instance Roosbeek et al. 1999; Herring et al. 2002). This signal clearly shows up in VLBI residuals. The apparent period oscillates between 430 and 460 days (Vondrák et al. 2005) and is most likely driven by diurnal atmospheric pressure variations (Gegout et al. 1998).

However, the time variation of the apparent period of this free mode does not give information on the deep Earth's structure, as it is related to the time variation of the excitation. The RFCN complex frequency is directly linked to the core flattening and to other deformability and coupling parameters. These parameters can be retrieved observationally considering the effect of the associated resonance on the forced nutations (see Mathews et al. 1991; Mathews et al. 2002). Roosbeek et al. (1999) find a resonant period between 431 and 434 days, starting from a 10-yr nutation series referred to the IERS 1996 model. The MHB model yields a period between 429.93 and 430.48 sidereal days, with a quality factor close to 20000 . Recent estimates from Vondrák et al. (2005) and Vondrák \& Ron (2006) using longer VLBI data sets confirm a very small deviation (0.11 day) against the MHB value. The time stability of these parameters has been addressed first in Roosbeek et al. (1999) and Hinderer et al. (2000). In both papers, the authors conclude that the small variations detected in the RFCN resonant period are not real but induced by the variable free mode excitation.

This study extends the works of Vondrák et al. (2005) and Vondrák \& Ron (2006) by checking the stability of the RFCN parameters with respect to existing VLBI data sets (Sect. 2.1) and to the perturbing atmospheric excitation (Sect. 2.2). Their time stability is addressed in Sect. 2.3.

\section{Determination of the RFCN frequency}

We use the time series of nutation offsets obtained from the analysis of dual frequency (S/X) multi-baseline 24-h VLBI experiments processed in a single ionosphere-free global solution. The data sets from the following analysis centers are considered: aus00006 (AUS, Geoscience Australia), bkg00007 (BKG, Bundesamt für Kartographie und Geodäsie, Germany), gsf2006d (GSF, NASA/Goddard Space Flight Center), usn2006c (USN, United States Naval Observatory), and the combinations ivs06q3e (IVS, midnight epoch) and ivsM6q3e (IVM, mid-session epoch) made by the International VLBI Service for Geodesy and Astrometry. In addition, we use our homemade series opa2007a (OPA) processed by the authors at the Paris Observatory IVS Analysis Center. All the above time series start on or before 1984 and run until or after 2006.5. Their 
Table 1. Characteristics of the VLBI solutions used in this work.

\begin{tabular}{|c|c|c|c|c|c|c|c|c|c|}
\hline & Time span & $\begin{array}{l}\text { No. } \\
\text { sess. }\end{array}$ & $\begin{array}{r}\text { Wrms } \\
X / Y \mu \text { as }\end{array}$ & $\mathrm{CRF}$ & $\begin{array}{l}\text { Elev. } \\
\text { cut-off }\end{array}$ & TRF & $\begin{array}{l}\text { Troposphere } \\
\text { zenith/grad }\end{array}$ & $\begin{array}{l}\text { A priori } \\
\text { prec/nut }\end{array}$ & Software \\
\hline AUS & 1983.10-2006.83 & 1591 & $201 / 197$ & $\begin{array}{l}\text { fixed to } \\
\text { ICRF Ext. } 2\end{array}$ & $\mathrm{n} / \mathrm{a}$ & $\begin{array}{c}\text { fixed to } \\
\text { VTRF } 2005\end{array}$ & $\begin{array}{c}\mathrm{VMF} \\
\text { random/constant }\end{array}$ & $\begin{array}{l}\text { IAU } 1976 \\
\text { IAU } 1980\end{array}$ & OCCAM 6.1 \\
\hline BKG & 1984.02-2006.83 & 3137 & $165 / 183$ & all global & $5^{\circ}$ & $\begin{array}{c}\text { global } \\
26 \text { sites }\end{array}$ & $\begin{array}{c}\text { NMF } \\
\text { 1-h/constant }\end{array}$ & $\begin{array}{c}\text { IAU } 1976 \\
\text { IERS } 1996\end{array}$ & $\begin{array}{c}\text { Calc } 9.3 \\
\text { Solve } 2003\end{array}$ \\
\hline GSF & 1979.59-2006.83 & 3954 & $159 / 168$ & $\begin{array}{c}686 \text { global } \\
192 \text { local }\end{array}$ & $5^{\circ}$ & $\begin{array}{l}\text { global } \\
35 \text { sites }\end{array}$ & $\begin{array}{c}\text { NMF } \\
20-\mathrm{min} / 6-\mathrm{h}\end{array}$ & IAU 2000 & $\begin{array}{c}\text { Calc } 10 \\
\text { Solve } 2006\end{array}$ \\
\hline OPA & 1984.02-2006.05 & 2807 & $153 / 157$ & $\begin{array}{l}533 \text { global } \\
202 \text { local }\end{array}$ & $6^{\circ}$ & $\begin{array}{l}\text { global } \\
35 \text { sites }\end{array}$ & $\begin{array}{c}\text { NMF } \\
20-\min / 6-h\end{array}$ & IAU 2000 & $\begin{array}{c}\text { Calc } 10 \\
\text { Solve } 2006\end{array}$ \\
\hline USN & 1979.59-2006.83 & 3524 & $162 / 164$ & $\begin{array}{c}686 \text { global } \\
192 \text { local }\end{array}$ & $5^{\circ}$ & $\begin{array}{l}\text { global } \\
35 \text { sites }\end{array}$ & $\begin{array}{c}\text { NMF } \\
\text { 20-min/6-h }\end{array}$ & IAU 2000 & $\begin{array}{c}\text { Calc } 10 \\
\text { Solve } 2006\end{array}$ \\
\hline IVS & 1979.59-2006.62 & 3465 & $178 / 206$ & & & & & & \\
\hline IVM & 1979.59-2006.62 & 3487 & $179 / 198$ & & & & & & \\
\hline
\end{tabular}

characteristics are reported in Table 1, along with some technical information. In BKG, GSF and USN, the no-net rotation (NNR) is applied to the 212 ICRF defining sources of Ma et al. (1998). In OPA, the NNR is applied to the 247 stable sources of Feissel-Vernier et al. (2006). The number of sites in the TRF column refers to the number of stations on which the net rotation/translation constraints on position and velocities are applied. VMF: Vienna mapping functions (Boehm \& Schuh 2004), NMF: Niell mapping functions (Niell 1996). The second line of the Troposphere column gives the time interval on which the troposphere zenith delay and the gradients are estimated. Calc 10 and Solve 2006 implement the NRO-based coordinate transformation (Capitaine et al. 2003).

Validating the current VLBI analysis strategy is not within the scope of this paper and will not be extensively discussed. However, one also has to keep in mind that VLBI global analysis allows the estimation of a large number of parameters, among which are the Earth orientation parameters (EOP) and station and radio source positions (either as global or local parameters). The analysis strategy aims to free EOP from reference frame effects. Different analysis strategies, based on the elimination of certain sessions or on the choice of different constraints on the celestial and/or terrestrial reference frames, will lead to differences in the final nutation series (see, e.g., Feissel-Vernier et al. 2006), and therefore in the geophysical parameter values that can be deduced.

\subsection{Determination of the RFCN parameters}

The method of retrieving the Earth's interior parameters through VLBI data is based on the transfer function that expresses the ratio between rigid and non rigid nutation amplitudes (resp. $\eta_{\mathrm{R}}$ and $\eta_{\mathrm{NR}}$ in the frequency domain):

$\eta_{\mathrm{NR}}(\sigma)=T\left(\sigma ; e \mid e_{\mathrm{R}}\right) \eta_{\mathrm{R}}(\sigma)$,

where $\sigma$ is the forcing frequency expressed in the Earth-fixed frame of reference, and $e$ and $e_{\mathrm{R}}$ are the true ellipticity of the Earth and its "rigid" value assumed in the construction of the rigid nutation theory. The formula of the transfer function corresponds to Eq. (42) of the MHB paper

$T\left(\sigma ; e \mid e_{\mathrm{R}}\right)=\frac{e_{\mathrm{R}}-\sigma}{e_{\mathrm{R}}+1} N_{0}\left[1+(1+\sigma)\left(Q_{0}+\sum_{\alpha=1}^{4} \frac{Q_{\alpha}}{\sigma-s_{\alpha}}\right)\right]$, with nominal values of the parameters taken in Table 6 of the same paper. The $s_{\alpha}$ are the eigenfrequencies of the three-layer Earth system. The RFCN frequency is $s_{2}$. For a given forcing frequency $\sigma$, the observed correction to the MHB transfer function is obtained by dividing the fitted amplitude by the rigid counterpart taken from Souchay et al. (1999), consistent with what was done in the MHB work. Then the observation equations are obtained by computing the partial derivative of $T\left(\sigma ; e \mid e_{\mathrm{R}}\right)$ with respect to $s_{2}$. Because the aim of the paper is to only analyze the sensitivity of the RFCN to different phenomena, all the other parameters in the transfer function are fixed to their MHB value.

Only VLBI observations between 1984 and 2006 are used hereafter in order to compare the series on their common time span. The nutation offsets are transformed, if necessary, in order to be expressed with respect to the MHB expansion in cartesian coordinates $(X, Y)$. We then proceed in a way similar to Vondrák et al. (2005). We use weighted least-squares estimates of prograde and retrograde amplitudes of the following forced nutation terms: 18.6-yr $(\Omega), 9.3-\mathrm{yr}(2 \Omega), 3.6-\mathrm{yr}(-2 l+2 F+\Omega)$, annual $\left(l^{\prime}\right)$, semi-annual $(2 F-2 D+2 \Omega)$, tri-annual $\left(l^{\prime}+2 F-2 D+2 \Omega\right)$, monthly $(l)$, and semi-monthly $(2 F+2 \Omega)$, jointly with a 2 nd order polynomial on each component, and a retrograde 460 -day term with free amplitude and phase accounting for the variable free motion. Note that a 18.6-yr is fitted to account for any mismodeling of this prominent nutation. However, the origin of the long term curvature observed in the VLBI residuals remains unclear and we choose to also fit a 2 nd order polynomial. Note also that the influence of the 18.6-yr term on the determination of the RFCN frequency remains relatively weak (see Dehant et al. 2003), so that any error on the estimation of the 18.6-yr amplitude would not significantly change the results of this study. The complex frequency of the RFCN is written $(2 \pi / P)(1+\mathrm{i} / 2 Q)$ where $P$ and $Q$ are real and stand for the period and the quality factor, respectively. With this convention, if $P$ is negative, a positive value of $Q$ indicates a damping. The analysis that principles are explained above is run for two different time spans: the 1984-2006 and 1990-2006. The latter time span, for which the 18.6-yr term is not estimated for obvious reasons, aims to suppress the early VLBI data to only keep data with higher quality. However, the remaining number of sessions is significantly smaller. The values of the resonant RFCN period $P$ and quality factor $Q$ are reported in Table 2, and in Fig. 1. In the table, the last line of each block gives the averaged of the above values. The "averaged" uncertainty is estimated as the standard 
Table 2. Averaged values of the resonant of the annual (A), semi-annual (SA) terms and resonant period $P$ and quality factor $Q$ of the retrograde free core nutation estimated from various VLBI series.

\begin{tabular}{|c|c|c|c|c|c|c|c|c|c|c|c|c|c|}
\hline & & \multirow{2}{*}{$\begin{array}{r}\text { A pro } \\
\mu \text { as }\end{array}$} & \multicolumn{3}{|c|}{ A ret } & SA pro & \multicolumn{3}{|c|}{ SA ret } & \multicolumn{2}{|l|}{$P$} & \multirow[t]{2}{*}{$Q$} & \multirow[b]{2}{*}{ \pm} \\
\hline & & & \pm & $\mu$ as & \pm & $\mu$ as & \pm & $\mu$ as & \pm & days & \pm & & \\
\hline \multirow{8}{*}{ 1984-2006 } & AUS & 17.0 & 5.7 & 20.5 & 5.7 & 22.8 & 5.7 & 12.4 & 5.7 & -430.13 & 0.17 & 25835 & 2397 \\
\hline & BKG & 3.6 & 2.8 & 52.5 & 2.8 & 27.2 & 2.8 & 32.9 & 2.8 & -429.84 & 0.20 & 27689 & 3080 \\
\hline & GSF & 15.8 & 2.8 & 32.2 & 2.8 & 11.0 & 2.8 & 13.0 & 2.8 & -430.24 & 0.07 & 24999 & 862 \\
\hline & IVS & 17.5 & 4.2 & 41.3 & 4.2 & 16.8 & 4.2 & 13.6 & 4.2 & -429.22 & 0.07 & 28917 & 1347 \\
\hline & IVM & 17.0 & 2.8 & 32.3 & 2.8 & 12.4 & 3.6 & 15.1 & 3.6 & -429.30 & 0.08 & 29796 & 1622 \\
\hline & OPA & 11.4 & 2.8 & 37.1 & 2.8 & 17.1 & 2.8 & 15.8 & 2.8 & -429.93 & 0.11 & 24169 & 1329 \\
\hline & USN & 9.4 & 2.8 & 33.1 & 2.8 & 9.0 & 2.8 & 16.0 & 2.8 & -429.89 & 0.09 & 25315 & 1205 \\
\hline & & 6.4 & 4.7 & 30.7 & 8.7 & 12.9 & 4.6 & 14.6 & 3.5 & -429.75 & 0.42 & 26090 & 1917 \\
\hline \multirow{8}{*}{ 1990-2006 } & AUS & 14.4 & 7.1 & 22.6 & 7.1 & 22.8 & 5.7 & 12.4 & 5.7 & -430.56 & 0.14 & 30731 & 2828 \\
\hline & BKG & 3.6 & 3.6 & 52.8 & 3.6 & 27.5 & 4.2 & 32.9 & 4.2 & -429.50 & 0.18 & 31329 & 3859 \\
\hline & GSF & 15.8 & 2.8 & 33.2 & 2.8 & 11.0 & 2.8 & 13.0 & 2.8 & -430.21 & 0.10 & 23610 & 1142 \\
\hline & IVS & 20.0 & 4.2 & 33.6 & 4.2 & 17.1 & 4.2 & 16.5 & 4.2 & -429.12 & 0.14 & 21681 & 1443 \\
\hline & IVM & 19.1 & 4.2 & 26.4 & 4.2 & 13.3 & 4.2 & 18.0 & 4.2 & -429.33 & 0.10 & 19071 & 821 \\
\hline & OPA & 12.4 & 2.8 & 37.1 & 2.8 & 17.0 & 2.8 & 15.5 & 2.8 & -430.26 & 0.13 & 26327 & 1887 \\
\hline & USN & 10.8 & 2.8 & 34.1 & 2.8 & 9.0 & 2.8 & 16.0 & 2.8 & -430.16 & 0.09 & 23016 & 974 \\
\hline & & 6.5 & 4.9 & 30.5 & 7.3 & 13.1 & 4.7 & 15.3 & 3.8 & -429.95 & 0.46 & 22173 & 2922 \\
\hline
\end{tabular}

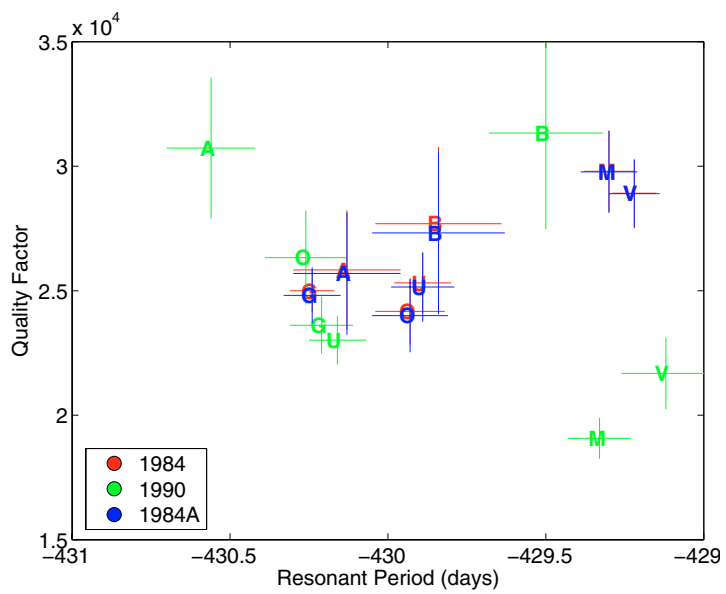

Fig. 1. Values of the resonant parameters as reported in Table 2 and after removing the atmospheric contribution (solution tagged 1984A). A: AUS, B: BKG, G: GSF, O: OPA, U: USN, V: IVS, M: IVM.

deviation of the relevant quantity (and not the averaged value of the individual uncertainties). In the figure, the uncertainties represent the formal error of the least-squares fit.

For the analysis run on 1984-2006, the resonant period appears to stay around -430 days within 0.4 days, a result consistent with the recent studies of Vondrák et al. (2005), wherein the estimate is made using a smoothed IVS combined series over a similar time span. The formal error reported is very small. The variability of the period noticed from one to the next VLBI data sets is larger by a factor of 3 . The quality factor remains around 26000 with a confidence interval around 2000. Running the analysis 1990-2006 produces a larger dispersion of the results.

\subsection{Contribution of the atmosphere}

Atmospheric angular momentum (AAM) time series are made available at the IERS Special Bureau for the Atmosphere (chaired by Salstein, at Atmospheric and Environmental Research, Inc., Mass., see Salstein et al. 1997), computed within the framework of the National Center for Environmental Prospect/National Center for Atmospheric

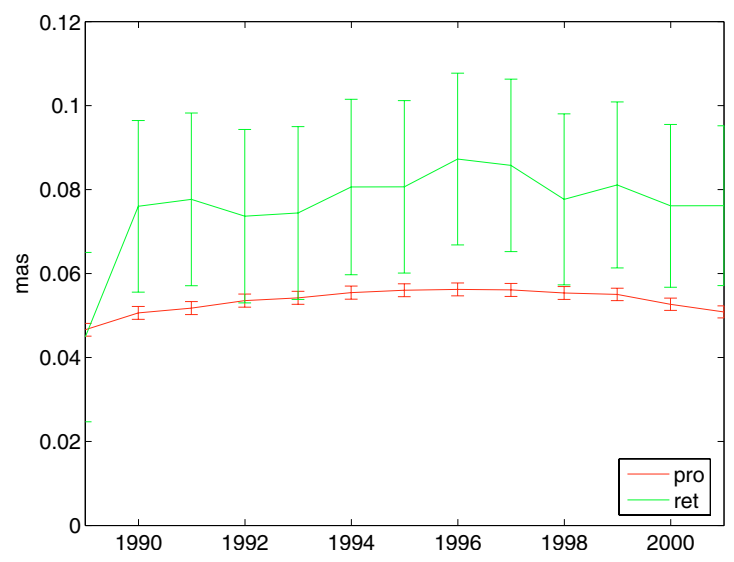

Fig. 2. Time variation of the prograde $\left(S_{1}\right)$ and retrograde $\left(\psi_{1}\right)$ atmospheric annual contributions to the nutation.

Research (NCEP/NCAR) Reanalysis project (Kalnay et al. 1993). We use the AAM computed without inverted barometer correction (i.e., we assume that, in the diurnal frequency band, oceans are not responding to atmospheric pressure variations). The atmospheric data were converted to the celestial frame and then resampled at 3-day intervals using a Gaussian interpolation, thereby removing the diurnal signal that is irrelevant here. The prograde $\left(S_{1}\right)$ and retrograde $\left(\psi_{1}\right)$ annual variations are then estimated over 1984-2006. The obtained amplitudes are transformed into a contribution to nutations using the transfer function as given in Bizouard et al. (1998). We find the following contributions to the prograde annual nutation: $-43 \pm 1$ and $26 \pm 1 \mu$ as, in phase and out of phase, respectively. For the retrograde annual nutation, we get $0 \pm 10$ and $64 \pm 10 \mu$ as, in phase and out of phase, respectively. These results agrees with Bizouard et al. (1998) or Yseboodt et al. (2002), where the authors already pointed out the extreme sensitivity of the estimates to AAM data set and the time span (see also similar remarks in the MHB paper, and in de Viron et al. 2005). The application of a 10 -yr sliding window displaced by 1 year (Fig. 2) shows that these amplitudes are stable in time over interannual scales. In the figure, the error bars are the result of amplifying the formal errors associated with the 

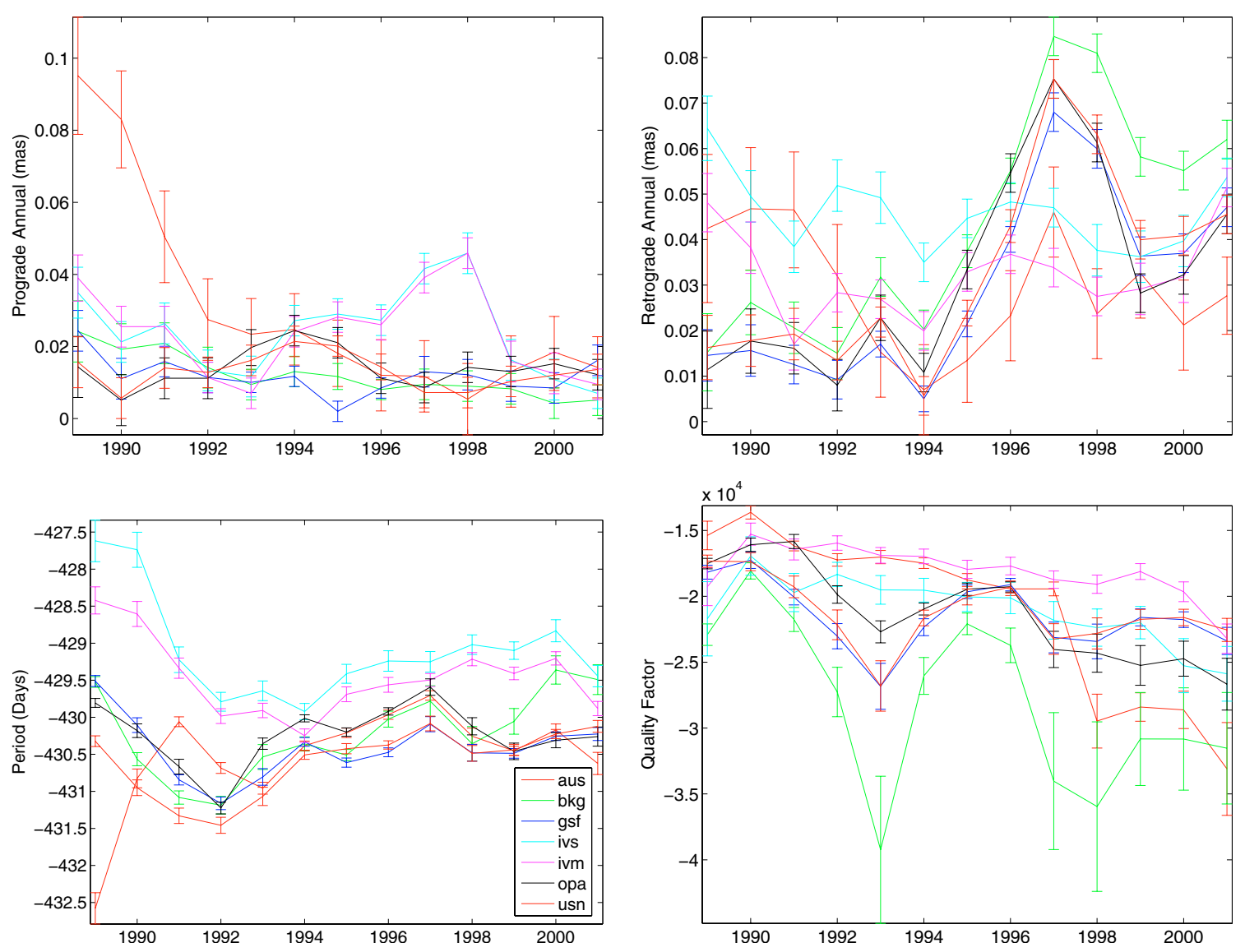

Fig. 3. Time variation of the prograde and retrograde amplitude in VLBI residuals and of the estimates of $P$ and $Q$ of the RFCN.

fit of $S_{1}$ and $\psi_{1}$. They appear larger for the retrograde part because of the resonance at the RFCN frequency in the formula of Bizouard et al. (1998). The mean amplitudes can be removed from those of the forced nutations estimated from VLBI in order to compute the RFCN parameters through atmosphere-free nutation offsets. The atmosphere-free RFCN parameters show a non-significant variation compared to the values when the atmosphere is not removed (see Fig. 1). Inconsistencies in the atmospheric models at the diurnal frequencies (see for instance Yseboodt et al. 2002; de Viron et al. 2005) will therefore not impact the determination of the RFCN parameters noticeably.

\subsection{Time variation}

To account for possible time instability, we apply a 10-yr slidingwindow on the data sets, displaced by 1 year, and we thus estimate "local" nutation amplitudes on shorter time intervals. (In this case the 18.6-yr wave is not estimated.) The width of the sliding-window is chosen to ensure that the free motion and the annual oscillation are decorrelated. Time variations of some parameters of interest are shown in Fig. 3. The analysis reveals differences between the various VLBI data sets. First of all, one sees that the magnitude of the retrograde annual nutation admits variations of $100 \%$ following the analysis center. For example the difference between GSF and AUS reaches $10 \mu$ as. For the prograde annual term, the AUS and the GSF series show variations that noticeably differ from all other data sets. Additionally, the curves for $P$ and $Q$ show a better time stability after 1994 , except for $Q$ deduced from AUS. The earlier years reveal instabilities that can cheat $P$ by 1 day and decrease $Q$ down to 15000 .
One also notes that, globally, all VLBI data sets give similar patterns. For instance, the retrograde annual term shows a maximum around 1997, which can be explained by the interaction with the retrograde free motion of period $\sim 430$ days. We investigated this interaction through a synthetic signal including an annual term, a retrograde free motion, and a Gaussian noise. Without free motion, the variation of the retrograde annual term is considerably lowered, and the remaining variation can be removed if the noise is turned off. The most likely annual amplitude is determined when the annual term and the free motion are in phase or when the free motion cancels out.

\section{Concluding remarks}

This work shows that the resonant RFCN parameters (period and quality factor) can be determined from recent VLBI observations with good reliability, as shown previously by Roosbeek et al. (1999), Herring et al. (2002), Vondrák et al. (2005), and Vondrák \& Ron (2006). In addition to these previous works, the stability of these parameters with respect to their time variability and the VLBI analysis strategy is checked. We found that

- the resonant period is stable within less than half a day when considering the time stability, assessed through a sliding window,

- the analysis strategy of VLBI observations, checked through several geodetic VLBI data sets using different sets of sessions, various constraints on reference frames, and a state-ofthe-art methodology, has also an impact of about half a day on the determination of the period, 
- the contribution of the atmospheric angular momentum, whose annual retrograde effect is enhanced by the resonance, is found to be negligible.

Nevertheless, we detected inconsistencies between series. Although small compared to the formal error on data, they should be investigated because they can reflect strengths or deficiencies in the analysis strategy, including theoretical models, software packages, or constraints applied to the reference frames. If not considered or corrected, they could have an impact on the precision of any future determination of the geophysical parameters.

Acknowledgements. The authors are grateful to Drs. O. de Viron \& A.-M. Gontier for useful advices and discussions. They also thank an anonymous referee for helping in improving the manuscript.

\section{References}

Bizouard, C., Brzeziński, A., \& Petrov, S. D. 1998, J. Geod., 72, 561

Boehm, J., \& Schuh, H. 2004, Geophys. Res. Lett., 31, L01603

Capitaine, N., Chapront, J., Lambert, S. B., \& Wallace, P. T. 2003, A\&A, 400, 1145
Dehant, V., Feissel-Vernier, M., de Viron, et al. 2003, J. Geophys. Res., 108 Feissel-Vernier, M., Ma, C., Gontier, A.-M., \& Barache, C. 2006, A\&A, 452, 1107

Fey, A. L., Ma, C., Arias, E. F., et al. 2004, AJ, 127, 3587

Gegout, P., Hinderer, J., Legros, H., et al. 1998, PEPI, 106, 337

Herring, T. A., Mathews, P. M., \& Buffett, B. A. 2002, J. Geophys. Res., 107

Hinderer, J., Boy, J.-P., Gegout, P., et al. 2000, PEPI, 117, 37

Kalnay, E., Kanamitsu, M., Kistler, R., et al. 1996, Bull. Amer. Meteor. Soc., 76, 437471

Ma, C., Arias, E. F., Eubanks, T. M., et al. 1998, AJ, 116, 516

MacMillan, D. S., \& Ma, C. 1999, Geophys. Res. Lett., 26, 919

Mathews, P. M., Buffett, B. A., Herring, T. A., \& Shapiro, I. I. 1991, J. Geophys. Res., 96, 8219

Mathews, P. M., Herring, T. A., \& Buffett, B. A. 2002, J. Geophys. Res., 107

Niell, A. 1996, J. Geophys. Res., 101, 3227

Roosbeek, F., Defraigne, P., Feissel, M., \& Dehant, V. 1999, Geophys. Res. Lett., 26,131

Salstein, D. A., Kann, D. M., Miller, A. J., \& Rosen, R. D. 1993, Bull. Amer. Meteorol. Soc., 74, 64

Souchay, J., Loysel, B., Kinoshita, H., \& Folgueira, M. 1999, A\&AS, 135, 111 de Viron, O., Schwarzbaum, G., Lott, F., \& Dehant, V. 2005, J. Geophys. Res., 110

Vondrák, J., Weber, R., \& Ron, C. 2005, A\&A, 444, 297

Vondrák, J., \& Ron, C. 2006, Acta Geodyn. Geomater., 3, 53

Yseboodt, M., de Viron, O., Chin, T. M., \& Dehant, V. 2002, J. Geophys. Res., 107 